Original Contribution

\title{
Correlation of clinicopathological features and LGR5 expression in colon adenocarcinoma
}

\author{
Koichi Sato ${ }^{\mathrm{a}}$, Takeshi Uehara ${ }^{\mathrm{b}, *}$, Mai Iwaya ${ }^{\mathrm{b}}$ Tomoyuki Nakajima $^{\mathrm{b}}$, Yusuke Miyagawa ${ }^{\mathrm{c}}$, \\ Hiroyoshi Ota ${ }^{\mathrm{b}, \mathrm{d}}$, Eiji Tanaka ${ }^{\mathrm{e}}$ \\ ${ }^{a}$ Department of Gastroenterology, National Hospital Organization, Shinshu Ueda Medical Center, Ueda, Japan \\ ${ }^{\mathrm{b}}$ Department of Laboratory Medicine, Shinshu University School of Medicine, Matsumoto, Japan \\ ${ }^{\text {c }}$ First Department of Surgery, Shinshu University School of Medicine, Matsumoto, Japan \\ ${ }^{\mathrm{d}}$ Department of Biomedical Laboratory Medicine, Shinshu University School of Medicine, Matsumoto, Japan \\ e Department for the Promotion of Regional Medicine, Shinshu University School of Medicine, Matsumoto, Japan
}

\section{A R T I C L E I N F O}

\section{Keywords:}

Leucine-rich repeat-containing G-protein-

coupled receptor 5

Tumor budding

RNA in situ hybridization

Colon adenocarcinoma

\begin{abstract}
A B S T R A C T
Colon cancer stem cells (CSCs) are closely related to tumorigenesis and treatment response, and LGR5 is currently the most robust and reliable CSC marker in colorectal cancer (CRC). However, LGR5 expression in CRC tumor budding (TB) is not well understood. We examined the clinicopathological and prognostic significance of LGR5 in CRC TB. LGR5 expression was evaluated by RNAscope, a newly developed RNA in situ hybridization technique, using a tissue microarray consisting of 55 patient samples of TB in colon adenocarcinoma (CA) selected from the medical archives at our hospital. Patients were stratified into negative and positive LGR5 expression groups. Tumor-infiltrating lymphocytes (TILs) and histological grade were lower in the LGR5-positive group compared with the LGR5-negative group $(P=.0407$ and $P=.0436$, respectively). There was no significant difference in overall survival between the LGR5-positive group and the LGR5-negative group (log-rank test, $P=.6931)$. LGR5 expression did not remain a predictor of prognosis in univariate analysis $(\mathrm{OR}=0.84$, 95\% CI: $0.33-2.02, P=.6928)$. LGR5 expression may be affected by TILs, which have been demonstrated to be associated with worse prognosis in the budding area of CA and is an important potential marker of prognosis.
\end{abstract}

\section{Introduction}

Colorectal cancer (CRC) is the second most common cause of cancer-related death worldwide [1]. Although early diagnosis and immediate surgery can cure patients with CRC, adjuvant chemotherapy is selected for high-risk CRC (stages II and III). However, stages II and III CRC are reported to recur in $30 \%$ and $40 \%$ of patients, respectively, despite adjuvant chemotherapy [2]. Since CRC recurrence causes a decrease in survival rate, development of a new treatment method is required.

The significance of cancer stem cells (CSCs) in cancer has been emphasized in recent years. CSCs comprise a small percentage of the total cancer tissue but are involved in self-renewal and metastasis and are resistant to chemotherapy $[3,4]$. LGR5 was identified by the lineage tracing method as the most promising stem cell marker in the colorectum and is considered a CSC marker in CRC [5-8]. It has also been reported as a CSC marker in several other tumors including those of the stomach [9-11], liver [12], and esophagus [13]. Several studies have analyzed LGR5 expression and prognosis $[14,15]$. There are several reports that high $L G R 5$ expression is associated with poor prognosis but this is controversial $[16,17]$.

The invasion front, especially tumor budding (TB) in CRC, is the forefront of tumor cell attack and host side defense and provides important prognostic information [18,19]. The clinicopathological and prognostic significance of LGR5 was analyzed in CRC TB using RNAscope, a newly described RNA in situ hybridization technique.

\section{Materials and methods}

\subsection{Patients}

This study enrolled 55 patients with colon adenocarcinoma (CA)

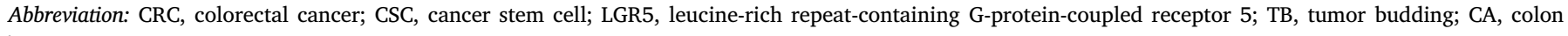
adenocarcinoma

* Corresponding author at: Department of Laboratory Medicine, Shinshu University School of Medicine, 3-1-1 Asahi, Matsumoto 390-8621, Japan.

E-mail address: tuehara@shinshu-u.ac.jp (T. Uehara).
} 

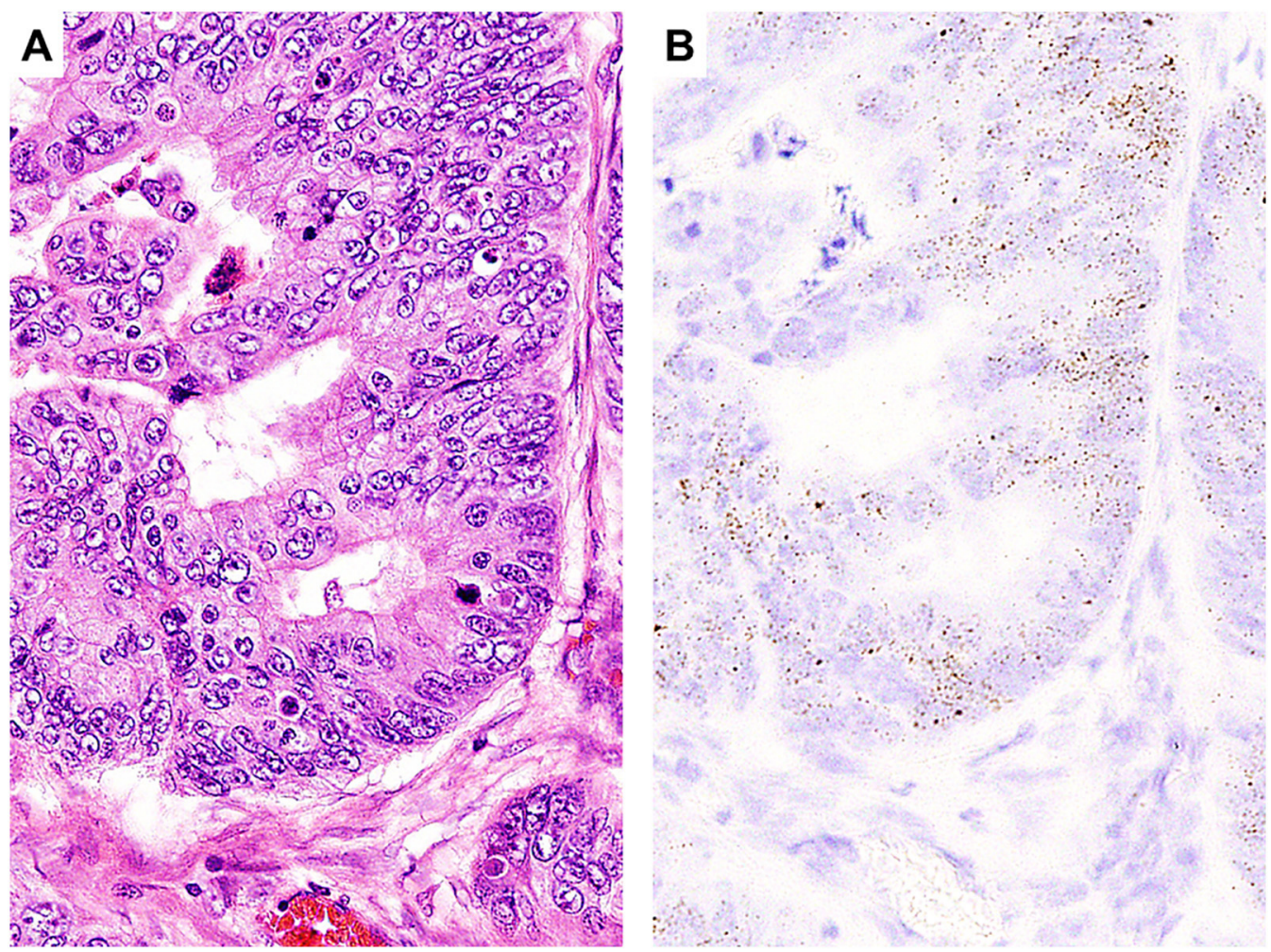

Fig. 1. Representative features of $L G R 5$ expression in CA (A and B). LGR5 expression was prominent in the gland-forming region recognized as low-grade CA. (A, HE; B, LGR5 RNAscope).

with TB who underwent surgical resection between 2010 and 2012 at Shinshu University School of Medicine, Matsumoto, Japan. Patients were followed up for at least 1 year. Clinicopathological data such as patient age and sex, pathological differentiation, inflammatory cell infiltration, vascular invasion, and TNM classification were obtained by retrospective review of medical charts and pathological records. Clinical stage and tumor differentiation were determined using the 8th edition of Union International Cancer Control (UICC) TNM staging system. Histological features of all specimens were confirmed by two pathologists (T.U. and H.O.). Overall survival (OS) was defined as the interval between the date of surgical resection and date of death or last follow up. This study was performed in accordance with the current ethical guidelines of the Declaration of Helsinki and the requirements of the Institutional Review Board of Shinshu University School of Medicine (approval No. 4088).

\subsection{Histopathology and immunohistochemistry}

All specimens were fixed in $20 \%$ formaldehyde and embedded in paraffin. Tumor blocks with sufficient tissue were selected to prepare a tissue microarray (TMA). The most representative region of the TB, defined as an area with a single cell or a detached group of tumor cells consisting of five cells or less, was selected based on the morphology of the hematoxylin and eosin (H\&E) stained slide. Tissue cores were punched out from each donor tumor block using thin-walled 3-mm stainless steel needles (Azumaya Medical Instruments Inc., Tokyo, Japan), and cores were arrayed in a recipient paraffin block. Serial sections of 4- $\mu \mathrm{m}$ thickness were cut from these blocks and stained with H\&E. TB was graded into Bd1 (0-4 buds), Bd2 (5-9 buds) and Bd3 ( $\geq 10$ buds) [20]. Furthermore, TB grade was categorized into low grade (Bd1 and Bd2) and high grade (Bd3). The score of inflammatory cell infiltration (tumor infiltrating lymphocytes, TILs) was measured in the budding area. The TIL score was assessed using a four-titer score and recorded as following; none: 0 , mild: 1 , moderate: 2 , and marked: 3
[21]. Furthermore, TIL score was categorized into low grade (score 0 and 1) and high grade (2 and 3).

\subsection{LGR5 RNA in situ hybridization}

Detection of LGR5 mRNA was performed using an RNAscope kit (Advanced Cell Diagnostics, Hayward, CA, USA) according to the manufacturer's instructions using unstained sample tissue slides. Briefly, tissue sections were pretreated with heating and protease application prior to hybridization with an LGR5-specific probe. The detailed procedure is described in a previous report [22]. Brown punctate dots present in the nucleus and/or cytoplasm indicated positive staining. LGR5 expression was quantified according to the 5-grade scoring system recommended by the manufacturer (no staining; 0, 1-3 dots/cell; $1+, 4-10$ dots/cell; 2+, 10-15 dots/cell; $3+$, and $>15$ dots/cell; $4+$ ) under a $20 \times$ objective lens (Olympus BX51). Furthermore, LGR5 mRNA expression was categorized into negative expression (grade 0 and $1+$ ) and positive expression $(2+, 3+$, and $4+$ ). We analyzed the relationship between LGR5 expression and clinicopathological data and prognosis in CA patients, with particular regard to OS rate.

\subsection{Statistical analysis}

For clinicopathological characteristics, categorical variables were expressed as a number. Pearson's chi-squared tests were adopted to test for differences between subgroups of patients. The OS rates of CA patients were calculated using the Kaplan-Meier method, and differences in those rates were compared by the log-rank test. The univariate and multivariate analyses for prognostic factors were performed using a Cox proportional hazard regression model. A $P$-value of $<0.05$ was considered significant. All statistical analyses were performed using JMP Statistics software version 13 (JMP, Tokyo, Japan). 

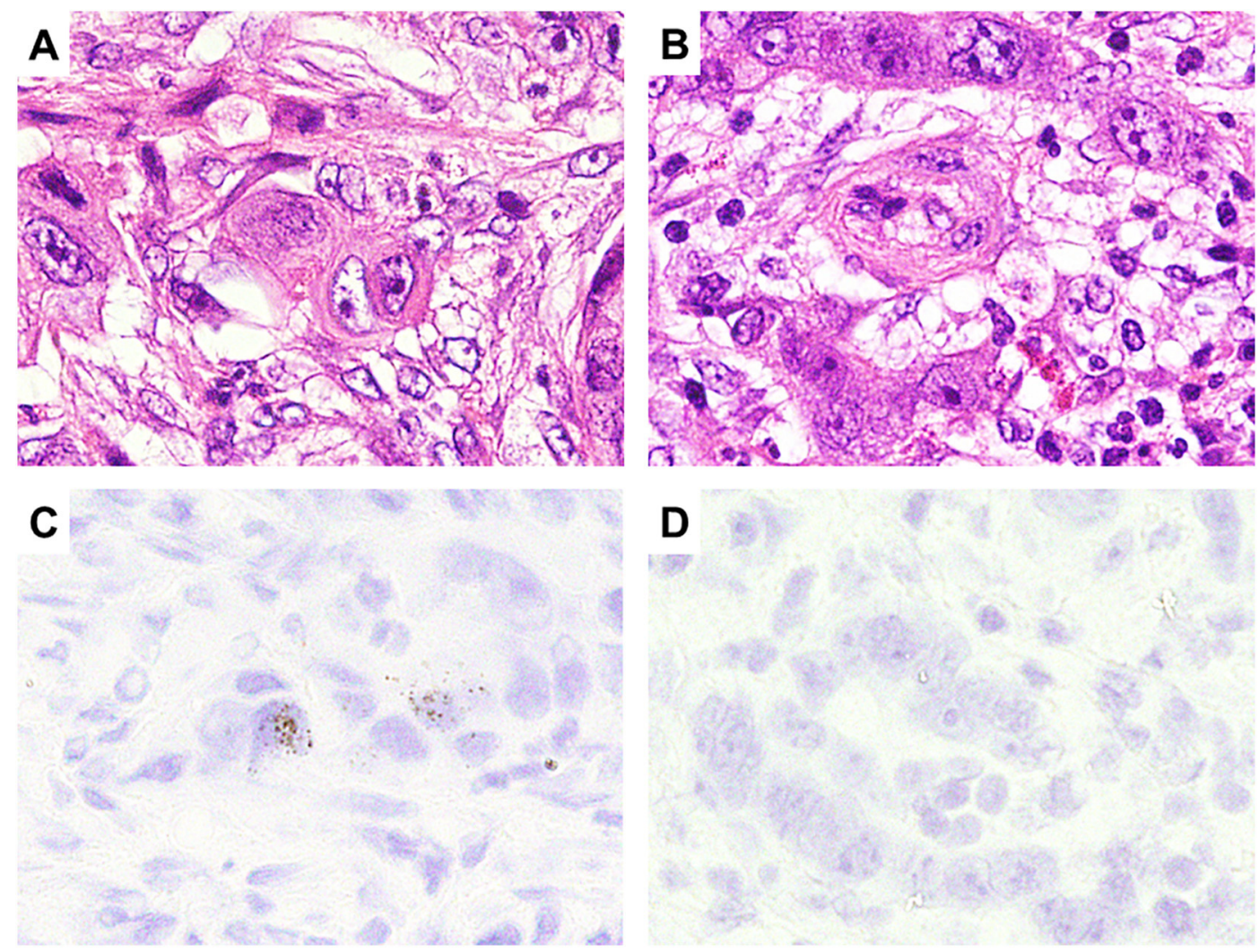

Fig. 2. LGR5 expression in TB. Representative features in TB with low-grade TILs (A) and high-grade TILs (B). In TB with low-grade TILs, high levels of LGR5 expression was observed (C). However, in TB with high-grade TILs, LGR5 expression was negative (D). (A and B, HE; C and D, LGR5 RNAscope).

\section{Results}

\subsection{LGR5 expression in CA}

In all cases, some LGR5-positive dots were detected in tumor cells, and there were a wide range of LGR5-positive cells. Fig. 1 shows representative images of LGR5 expression in CA. Expression of LGR5 was prominent in the gland-forming region. In TB, 35 cases showed some LGR5-positive dots over a wide range, but there were no positive findings in 20 cases. Twenty-six cases were classified into an LGR5positive group (Fig. 2A, C) and 29 cases were classified into an LGR5negative group (Fig. 2B, D).

\subsection{LGR5 expression and clinicopathological characteristics in CA}

The clinicopathological characteristics of patients with CA are described in Table 1. In the LGR5-positive group, TILs occurred in lower numbers than in the LGR5-negative group ( $P=.0407)$. In the LGR5positive group, histological grade was lower than that of the LGR5negative group $(P=.0436)$. There was no significant difference between the LGR5-positive group and the LGR5-negative group in terms of age, sex, vascular invasion, or TNM stage.

\subsection{Prognostic value of LGR5 in CA}

We assessed the prognostic value of LGR5 expression in CA by Kaplan-Meier analysis and the log-rank test. The median OS rate for the study patients was 1839 (range: 949-2379) days. There was no significant difference in OS between CA cases in the LGR5-positive group (median OS, 1988 [range, 972.25-2545.5] days) and the LGR5-negative group (median OS, 1837 [range; 887-2188] days) (log rank test, $P=.6931)$.

LGR5 expression did not remain a predictor of prognosis in
Table 1

LGR5 expression and clinicopathological characteristics in CA.

\begin{tabular}{|c|c|c|c|c|}
\hline \multirow[t]{2}{*}{ Factors } & \multirow[t]{2}{*}{$\mathrm{n}$} & \multicolumn{2}{|l|}{ LGR5 expression } & \multirow[t]{2}{*}{$P$-value } \\
\hline & & Positive $(n=26)$ & Negative $(n=29)$ & \\
\hline Age & & & & 0.5039 \\
\hline$>70$ years & 27 & 14 & 13 & \\
\hline$\leq 70$ years & 28 & 12 & 16 & \\
\hline Sex & & & & 0.1416 \\
\hline Male & 29 & 11 & 18 & \\
\hline Female & 26 & 15 & 11 & \\
\hline TILs & & & & 0.0407 \\
\hline High & 27 & 9 & 18 & \\
\hline Low & 28 & 17 & 11 & \\
\hline Histological grade & & & & 0.0436 \\
\hline High & 29 & 10 & 19 & \\
\hline Low & 26 & 16 & 10 & \\
\hline Vascular invasion & & & & 0.9444 \\
\hline High & 32 & 15 & 17 & \\
\hline Low & 23 & 11 & 12 & \\
\hline Tumor budding grade & & & & 0.6093 \\
\hline High & 10 & 4 & 6 & \\
\hline Low & 45 & 22 & 23 & \\
\hline TNM stage & & & & 0.4394 \\
\hline I-II & 22 & 9 & 13 & \\
\hline III-IV & 33 & 17 & 16 & \\
\hline
\end{tabular}

univariate analysis (OR $=0.84,95 \%$ CI: $0.33-2.02, P=.6928)$ (Table 2).

\section{Discussion}

Although there is no direct involvement of LGR5 expression on OS in TB, the correlation between LGR5 expression and TILs may indicate that high LGR5 expression confers a poor prognosis, considering that 
Table 2

Univariate analyses for prognostic factors of CA.

\begin{tabular}{lll}
\hline \multirow{2}{*}{ Factors } & \multicolumn{2}{l}{ Univariate analysis } \\
\cline { 2 - 3 } & OR $(95 \% \mathrm{CI})$ & $P$-value \\
\hline Age: $>$ 70 years vs $\leq 70$ years & $2.59(1.06-6.92)$ & 0.0369 \\
Sex: male vs. female & $2.84(1.13-8.03)$ & 0.0251 \\
Histological grade: low vs. high & $0.52(0.20-8.03)$ & 0.1574 \\
TIL: low grade vs. high grade & $3.46(1.34-10.66)$ & 0.0095 \\
Vascular invasion: absent vs. present & $0.72(0.27-1.76)$ & 0.4771 \\
Tumor budding grade: low vs. high & $0.41(0.16-1.16)$ & 0.0896 \\
TNM stage: I-II vs. III-IV & $0.54(0.19-1.35)$ & 0.1928 \\
LGR5 expression: positive vs. negative & $0.84(0.33-2.02)$ & 0.6928 \\
\hline
\end{tabular}

low-grade TIL is involved in poor prognosis [23]. It is reported that TILs are related to microsatellite instability-high (MSI-H) CRC [24]. MSI-H CRC may be classified into one category of the disease called hypermutated type. Therefore, it may be necessary to increase the number of cases and examine LGR5 expression in the presence or absence of TILs. Evaluation of TB in CRC is performed using various techniques. Although TB grade showed no significant association with OS in our study, there are several reports on the number of tumor cells and the degree of differentiation in TB, as well as the relationship between the type of TB and prognosis [25,26].

The expression of various markers in TB has been analyzed and there have been several reports of CSC marker expression in recent years. In the TB the decreased expression of CD44, which is known to be a representative stem cell marker, has been reported to indicate a poor prognosis [27]. Although another stem cell marker, CD133, exhibits negative or weak positivity in TB, no association with clinicopathological data has been reported $[28,29]$.

LGR5 is recognized not only as the most promising stem cell marker in the colorectum but also as a CSC marker in CA [5-8]. Several studies on LGR5 expression and clinicopathological analysis in CA have been reported, but there are few reports on TB [30,31]. Zheng et al. reported that TB is not related to LGR5 expression and distant metastasis [30]. Moreover, the relationship between distant metastasis and LGR5 expression is controversial in other tissues [7,30,32]. LGR5 has been reported to be associated with markers that promote epithelial-mesenchymal transition (EMT), which promotes cancer metastasis [33]. Although the expression of many genes related to EMT has been reported in tumor cells of TB [34], Jang et al. reported that attenuation of LGR5 expression in TB correlates with promotion of EMT [31]. In our study, there was no significant difference between LGR5 expression and prognosis. However, in LGR5-positive cases, the promotion of EMT was involved in metastasis and may result in poor prognosis.

In TB, the LGR5-positive group with significantly low-grade CA might have been affected by the LGR5 expression levels in low-grade CA tissue with gland formation. In our present study, LGR5 expression was more commonly identified in low-grade CA with gland formation. The relationship between tumor grade and LGR5 expression level is controversial [14,30-32,35]. Many of these reports used immunohistochemistry to evaluate LGR5. However, in recent years, RNA in situ hybridization has been reported to reveal strong expression of LGR5 in low-grade CA with gland formation [31]. This difference may be because the LGR5 expression site differs between the two methods. However, because the reliability of immunohistochemistry for LGR5 is questionable [31], RNA in situ hybridization may be more reliable.

Declining TIL numbers in the LGR5-positive group may be a protective function of stem cells. LGR5 expression may be affected by TILs in the budding area of CA. There is no report on the relationship between LGR5 and TILs despite there being reports on stem cell markers in other cancer types. The expression of ALDH1, which is considered to be a CSC marker in breast cancer, is high in TIL-positive cases [36]. Since ALDH1 is also an enzyme involved in detoxification, its function differs from that of LGR5 [37]. Therefore, the reversal in the expression levels of LGR5 and ALDH1 in TILs may be due to differences in carcinomas and in the function of CSC markers.

A limitation of our study is that only LGR5 mRNA expression was evaluated and thus the functional analysis of LGR5 protein expression in cultured cells is warranted. It may also be necessary to analyze a larger number of cases in a long-term follow-up cohort study.

In conclusion, this study showed that high LGR5 expression in TB, which is more frequent in low-histological grade CA, is involved in lowgrade TIL, which has been demonstrated to be associated with poor prognosis, and thus LGR5 expression in TB may have important implications in CSC-targeted therapy.

\section{Acknowledgements}

We are grateful to Yukihoro Kobayashi, Masanobu Momose, Yasuyo Shimojo, Naoko Ogiwara, Mieko Horikawa, Akiko Inamura, Chitoshi Arai, Yasuhiro Kinugawa, Marina Nuno, and Souya Ogasawara at Shinshu University Hospital for their excellent technical assistance. This study was supported by the Hokuto Foundation for Bioscience, Japan (Grant Award to T.U.). We thank H. Nikki March, PhD, from Edanz Group (www.edanzediting.com/ac) for editing a draft of this manuscript. Part of this submission was presented at Annual Meeting USCAP 2019, March 16-21, National Harbor, Maryland.

\section{Declaration of competing interest}

None declared.

\section{References}

[1] Bray F, Ferlay J, Soerjomataram I, et al. Global cancer statistics 2018: GLOBOCAN estimates of incidence and mortality worldwide for 36 cancers in 185 countries. CA Cancer J Clin 2018;68:394-424.

[2] Dy GK, Hobday TJ, Nelson G, et al. Long-term survivors of metastatic colorectal cancer treated with systemic chemotherapy alone: a North Central Cancer Treatment Group review of 3811 patients, N0144. Clin Colorectal Cancer 2009;8:88-93.

[3] Reya T, Morrison SJ, Clarke MF, et al. Stem cells, cancer, and cancer stem cells. Nature 2001;414:105-11.

[4] Dean M, Fojo T, Bates S. Tumour stem cells and drug resistance. Nat Rev Cancer 2005;5:275-84.

[5] Barker N, van Es JH, Kuipers J, et al. Identification of stem cells in small intestine and colon by marker gene Lgr5. Nature 2007;449:1003-7.

[6] Ziskin JL, Dunlap D, Yaylaoglu M, et al. In situ validation of an intestinal stem cell signature in colorectal cancer. Gut 2013;62:1012-23.

[7] He S, Zhou H, Zhu X, et al. Expression of Lgr5, a marker of intestinal stem cells, in colorectal cancer and its clinicopathological significance. Biomed Pharmacother 2014;68:507-13.

[8] Uchida H, Yamazaki K, Fukuma M, et al. Overexpression of leucine-rich repeatcontaining G protein-coupled receptor 5 in colorectal cancer. Cancer Sci 2010;101:1731-7.

[9] Simon E, Petke D, Boger C, et al. The spatial distribution of LGR5 + cells correlates with gastric cancer progression. PLoS One 2012;7:e35486.

[10] Yamanoi K, Fukuma M, Uchida H, et al. Overexpression of leucine-rich repeatcontaining G protein-coupled receptor 5 in gastric cancer. Pathol Int 2013;63:13-9.

[11] Jang BG, Lee BL, Kim WH. Prognostic significance of leucine-rich-repeat-containing G-protein-coupled receptor 5, an intestinal stem cell marker, in gastric carcinomas. Gastric Cancer 2016;19:767-77.

[12] Fukuma M, Tanese K, Effendi K, et al. Leucine-rich repeat-containing G proteincoupled receptor 5 regulates epithelial cell phenotype and survival of hepatocellular carcinoma cells. Exp Cell Res 2012;319:113-21.

[13] Becker L, Huang Q, Mashimo H. Lgr5, an intestinal stem cell marker, is abnormally expressed in Barrett's esophagus and esophageal adenocarcinoma. Dis Esophagus 2010;23:168-74.

[14] Jiang Y, Li W, He X, et al. Lgr5 expression is a valuable prognostic factor for colorectal cancer: evidence from a meta-analysis. BMC Cancer 2016;16:12.

[15] Han Y, Xue X, Jiang M, et al. LGR5, a relevant marker of cancer stem cells, indicates a poor prognosis in colorectal cancer patients: a meta-analysis. Clin Res Hepatol Gastroenterol 2015;39:267-73.

[16] Takahashi H, Ishii H, Nishida N, et al. Significance of Lgr5(+ve) cancer stem cells in the colon and rectum. Ann Surg Oncol 2011;18:1166-74.

[17] Martin ML, Zeng Z, Adileh M, et al. Logarithmic expansion of LGR5( + ) cells in human colorectal cancer. Cell Signal 2018;42:97-105.

[18] Zlobec I, Lugli A. Invasive front of colorectal cancer: dynamic interface of pro-/antitumor factors. World J Gastroenterol 2009;15:5898-906.

[19] Konishi T, Shimada Y, Lee LH, et al. Poorly differentiated clusters predict colon 
cancer recurrence: an in-depth comparative analysis of invasive-front prognostic markers. Am J Surg Pathol 2018;42:705-14.

[20] Lugli A, Kirsch R, Ajioka Y, et al. Recommendations for reporting tumor budding in colorectal cancer based on the International Tumor Budding Consensus Conference (ITBCC) 2016. Mod Pathol 2017;30:1299-311.

[21] Ropponen KM, Eskelinen MJ, Lipponen PK, et al. Prognostic value of tumour-infiltrating lymphocytes (TILs) in colorectal cancer. J Pathol 1997;182:318-24.

[22] Ukpo OC, Flanagan JJ, Ma XJ, et al. High-risk human papillomavirus E6/E7 mRNA detection by a novel in situ hybridization assay strongly correlates with p16 expression and patient outcomes in oropharyngeal squamous cell carcinoma. Am J Surg Pathol 2011;35:1343-50.

[23] Idos GE, Kwok J, Bonthala N, et al. The prognostic implications of tumor infiltrating lymphocytes in colorectal cancer: a systematic review and meta-analysis. Sci Rep 2020;10:3360

[24] Williams DS, Mouradov D, Jorissen RN, et al. Lymphocytic response to tumour and deficient DNA mismatch repair identify subtypes of stage II/III colorectal cancer associated with patient outcomes. Gut 2019;68:465-74.

[25] Wang LM, Kevans D, Mulcahy H, et al. Tumor budding is a strong and reproducible prognostic marker in T3N0 colorectal cancer. Am J Surg Pathol 2009;33:134-41.

[26] Lee VWK, Chan KF. Tumor budding and poorly-differentiated cluster in prognostication in stage II colon cancer. Pathol Res Pract 2018;214:402-7.

[27] Choi JE, Bae JS, Kang MJ, et al. Expression of epithelial-mesenchymal transition and cancer stem cell markers in colorectal adenocarcinoma: clinicopathological significance. Oncol Rep 2017;38:1695-705.

[28] Li CY, Li BX, Liang Y, et al. Higher percentage of CD133 + cells is associated with poor prognosis in colon carcinoma patients with stage IIIB. J Transl Med 2009;7:56.

[29] Horst D, Kriegl L, Engel J, et al. CD133 expression is an independent prognostic marker for low survival in colorectal cancer. Br J Cancer 2008;99:1285-9.

[30] Zheng Z, Yu H, Huang Q, et al. Heterogeneous expression of Lgr5 as a risk factor for focal invasion and distant metastasis of colorectal carcinoma. Oncotarget 2018;9:30025-33.

[31] Jang BG, Kim HS, Chang WY, et al. Expression profile of LGR5 and its prognostic significance in colorectal cancer progression. Am J Pathol 2018;188:2236-50.

[32] Wu XS, Xi HQ, Chen L. Lgr5 is a potential marker of colorectal carcinoma stem cells that correlates with patient survival. World J Surg Oncol 2012;10:244.

[33] Zhang S, Han X, Wei B, et al. RSPO2 enriches LGR5(+) spheroid colon cancer stem cells and promotes its metastasis by epithelial-mesenchymal transition. Am J Transl Res 2016;8:354-64.

[34] De Smedt L, Palmans S, Andel D, et al. Expression profiling of budding cells in colorectal cancer reveals an EMT-like phenotype and molecular subtype switching. Br J Cancer 2017;116:58-65.

[35] Hsu HC, Liu YS, Tseng KC, et al. Overexpression of Lgr5 correlates with resistance to 5-FU-based chemotherapy in colorectal cancer. Int J Colorectal Dis 2013;28:1535-46.

[36] Polonia A, Pinto R, Cameselle-Teijeiro JF, et al. Prognostic value of stromal tumour infiltrating lymphocytes and programmed cell death-ligand 1 expression in breast cancer. J Clin Pathol 2017;70:860-7.

[37] Tomita H, Tanaka K, Tanaka T, et al. Aldehyde dehydrogenase 1A1 in stem cells and cancer. Oncotarget 2016;7:11018-32. 